\title{
THE GENERALIZED WITT MODULAR LIE SUPERALGEBRA OF CARTAN TYPE
}

\author{
YAN-QIN DONG ${ }^{凶}$, YONG-ZHENG ZHANG and ANGELO EBONZO
}

(Received 28 April 2009; accepted 11 August 2011)

Communicated by J. Du

\begin{abstract}
We construct the generalized Witt modular Lie superalgebra $\tilde{W}$ of Cartan type. We give a set of generators for $\tilde{W}$ and show that $\tilde{W}$ is an extension of a subalgebra of $\tilde{W}$ by an ideal $\bar{J}$. Finally, we describe the homogeneous derivations of Z-degree of $\tilde{W}$ and we determine the derivation superalgebra of $\tilde{W}$.
\end{abstract}

2010 Mathematics subject classification: primary 17B50.

Keywords and phrases: modular Lie superalgebras, Z-graded Lie superalgebras, derivation superalgebras.

\section{Introduction}

The theory of Lie superalgebras over a field of characteristic zero is very well developed (see, for example, $[4,5,10])$. But the same is not true for modular Lie superalgebras. For instance, the classification of finite-dimensional simple modular Lie superalgebras is not yet complete. As far as we know, the $(p, 2 p)$-structure for modular Lie superalgebras (analogous to $p$-mappings for modular Lie algebras) was introduced by Kochetkov and Leites [6]. Later, Petrogradski [9] studied restricted enveloping algebras for modular Lie superalgebras, and Farnsteiner [2] worked on Frobenius extensions and restricted modular Lie superalgebras. In 1997, Zhang [13] constructed four classes of finite-dimensional Cartan type modular Lie superalgebras $X(m, n, \boldsymbol{t})$ and studied their simplicity and restrictiveness, where $X$ is one of the algebras $W, S, H$ or $K$.

Derivation algebras of Lie algebras play an important role in the study of properties of Lie algebras such as filtrations and automorphism groups. Celousov [1] and Petrogradski [9] investigated derivation algebras of Cartan type modular Lie algebras. Derivation superalgebras of Cartan type modular Lie superalgebras are becoming a

This work was supported by National Science Foundation of China Funded Projects (Nos. 10871057 and 10701019).

(C) 2011 Australian Mathematical Publishing Association Inc. 1446-7887/2011 \$16.00 
subject of interest in the structure theory of Lie superalgebras. Due to the prime characteristic and superstructure of Lie superalgebras, their derivation superalgebras are harder to determine. Despite this, the derivation superalgebras of $\mathrm{W}, \mathrm{S}, \mathrm{H}, \mathrm{K}, \mathrm{HO}$ and $K O$ have been determined (see [3, 7, 8, 12, 15]).

Our work is motivated by the results and methods for Lie algebras and Lie superalgebras and is based on certain results on modular Lie algebras and Lie superalgebras of Cartan type (see [11, 14, 15]). The paper is organized as follows. In Section 2, we construct the finite-dimensional generalized Witt modular Lie superalgebra. In Section 3 , we give a set of generators for $\tilde{W}$ and show that $\tilde{W}$ is not simple; moreover, we show that $\tilde{W}$ is an extension of a subalgebra of $\tilde{W}$ by the ideal $\bar{J}$. In Section 4 , we establish some technical lemmas and determine the derivation superalgebra of $\tilde{W}$.

\section{Basics and construction}

Throughout this paper, $F$ denotes a field of characteristic $p$, greater than 2 , and $Z_{2}=\{\overline{0}, \overline{1}\}$ denotes the field of two elements. We use the notation $N$ and $N_{0}$ to stand for the sets of positive integers and nonnegative integers, respectively. For $n \in N$ and $\alpha=\left(\alpha_{1}, \alpha_{2}, \ldots, \alpha_{n}\right) \in N_{0}^{n}$, we define $|\alpha|=\sum_{i=1}^{n} \alpha_{i}$.

Let $O(n)$ denote the divided power algebra with an $F$-basis $\left\{x^{(\alpha)} \mid \alpha \in N_{0}^{n}\right\}$. Put $\boldsymbol{t}=\left(t_{1}, t_{2}, \ldots, t_{n}\right) \in N_{0}^{n}$ and $\pi_{i}=p^{t_{i}}-1$, where $i=1,2, \ldots, n$. Let

$$
A(\boldsymbol{t}):=\left\{\alpha=\left(\alpha_{1}, \alpha_{2}, \ldots, \alpha_{n}\right) \in N_{0}^{n} \mid 0 \leq \alpha_{i} \leq \pi_{i}\right\}
$$

Then

$$
O(n, t):=\operatorname{span}_{F}\left\{x^{(\alpha)} \mid \alpha \in A(t)\right\}
$$

is a finite-dimensional subalgebra of $O(n)$.

Let $\Lambda(q)$ denote the Grassmann superalgebra over $F$ in $q$ variables $x_{n+1}, x_{n+2}, \ldots$, $x_{r}$, where $r=n+q$. In order to shorten the notation for the Grassmann superalgebra, we put

$$
B_{k}=\left\{\left\langle i_{1}, i_{2}, \ldots, i_{k}\right\rangle \mid n+1 \leq i_{1}<i_{2}<\cdots<i_{k} \leq r\right\}
$$

and $B(q)=\bigcup_{k=0}^{q} B_{k}$, where $B_{0}=\emptyset$. When $\mu=\left\langle i_{1}, i_{2}, \ldots, i_{k}\right\rangle \in B_{k}$, we define $|\mu|=k$, $\{\mu\}=\left\{i_{1}, i_{2}, \ldots, i_{k}\right\}$ and $x^{\mu}=x_{i_{1}} x_{i_{2}} \cdots x_{i_{k}}$, where we adopt the conventions that $|\emptyset|:=0$ and $x^{\emptyset}=1$. Then the set $\left\{x^{\mu} \mid \mu \in B(q)\right\}$ is an $F$-basis of $\Lambda(q)$.

We now fix two positive integers $m_{1}$ and $m_{2}$. We write $m:=m_{1}+m_{2}, s:=r+m_{1}$ and $s_{1}:=s+m_{2}$. Let

$$
Q(m)=F\left[y_{r+1}, \ldots, y_{s}, y_{s+1}, \ldots, y_{s_{1}}\right]
$$

be the truncated polynomial algebra such that $y_{i}^{p}=1$ for $i=r+1, \ldots, s_{1}$. We let $\Pi=\{0,1, \ldots, p-1\}$ denote the prime subfield of $F$ and write $H:=\Pi^{m}$. For every element $\lambda=\left(\lambda_{r+1}, \ldots, \lambda_{s_{1}}\right) \in H$, we define $y^{\lambda}=\prod_{r+1}^{s_{1}} y_{i}^{\lambda_{i}}$. Then $Q(m)$ has an $F$-basis $\left\{y^{\lambda} \mid \lambda \in H\right\}$. The tensor product

$$
G:=O(n, t) \otimes \Lambda(q) \otimes Q(m)
$$


is an associative superalgebra with a $Z_{2}$-gradation induced by the standard $Z_{2}$-gradation of $\Lambda(q)$. For $f \in O(n, t), g \in \Lambda(q)$ and $h \in Q(m)$, we write $f g h$ for $f \otimes g \otimes h$. Then

$$
\left\{x^{(\alpha)} x^{\mu} y^{\lambda} \mid \alpha \in A(\boldsymbol{t}), \mu \in B(q), \lambda \in H\right\}
$$

is an $F$-basis for $G$.

Let $E=\langle n+1, \ldots, r\rangle$ and $\pi=\left(\pi_{1}, \ldots, \pi_{n}\right)$. Clearly, $E \in B(q)$ and $\pi \in A(t)$. For convenience, we write $Y_{0}=\{1, \ldots, n\}, Y_{1}=\{n+1, \ldots, r\}$ and $Y_{2}=\{r+1, \ldots, s\}$. Let $Y=Y_{0} \cup Y_{1}$ and $S=Y \cup Y_{2}$. When $i \in Y_{0}$ and $\varepsilon_{i}=\left(\delta_{i 1}, \delta_{i 2}, \ldots, \delta_{i n}\right)$, we abbreviate $x^{\left(\varepsilon_{i}\right)}$ to $x_{i}$. When $i=r+1, \ldots, s_{1}$ and $\bar{\varepsilon}_{i}=\left(\delta_{i(r+1)}, \delta_{i(r+2)}, \ldots, \delta_{i s_{1}}\right)$, we abbreviate $y^{\bar{\varepsilon}_{i}}$ to $y_{i}$.

Let $D_{1}, D_{2}, \ldots, D_{s}$ be the linear transformations of $G$ such that

$$
D_{i}\left(x^{(\alpha)} x^{\mu} y^{\lambda}\right)= \begin{cases}x^{\left(\alpha-\varepsilon_{i}\right)} x^{\mu} y^{\lambda} & \text { if } i \in Y_{0}, \\ x^{(\alpha)} \partial_{i}\left(x^{\mu}\right) y^{\lambda} & \text { if } i \in Y_{1}, \\ \lambda_{i} x^{(\alpha)} x^{\mu} y^{\lambda} & \text { if } i \in Y_{2} .\end{cases}
$$

Here $\partial_{i}$ is the derivation of $\Lambda(q)$ such that $\partial_{i}\left(x_{j}\right)=\delta_{i j}$ for $i, j \in Y_{1}$. Then $D_{1}$, $D_{2}, \ldots, D_{s}$ are derivations of the superalgebra $G$. Let

$$
\tilde{W}(n, \boldsymbol{t}, q, m)=\left\{\sum_{i=1}^{s} f_{i} D_{i} \mid f_{i} \in G\right\} .
$$

For a superalgebra (or a superspace) $L=L_{\overline{0}} \oplus L_{\overline{1}}$, we write $\mathrm{h}(L)=L_{\overline{0}} \cup L_{\overline{1}}$ for the set of all $Z_{2}$-homogeneous elements of $L$ and write $|x|$ for the $Z_{2}$-degree of a given homogeneous element $x$. It is clear that $\left|D_{i}\right|=\tilde{\imath}$, where

$$
\tilde{\imath}= \begin{cases}\overline{0} & \text { if } i \in Y_{0} \cup Y_{2}, \\ \overline{1} & \text { if } i \in Y_{1} .\end{cases}
$$

Set

$$
\tilde{W}_{\gamma}=\operatorname{span}_{F}\left\{x^{(\alpha)} x^{\mu} y^{\lambda} D_{i} \mid \overline{|\mu|}+\tilde{\imath}=\gamma\right\}
$$

for $\gamma \in Z_{2}$. Then $\tilde{W}=\bigoplus_{\gamma \in Z_{2}} \tilde{W}_{\gamma}$. The following formula holds in $\tilde{W}(n, \boldsymbol{t}, q, m)$ :

$$
\left[f D_{i}, g D_{j}\right]=f D_{i}(g) D_{j}-(-1)^{\left|f D_{i} \| g D_{j}\right|} g D_{j}(f) D_{i}
$$

for $f, g \in \mathrm{h}(G)$ and $i, j \in S$. It follows that $\tilde{W}(n, t, q, m)$ is a finite-dimensional Lie superalgebra contained in $\operatorname{der} G$. We abbreviate $\tilde{W}(n, \boldsymbol{t}, q, m)$ to $\tilde{W}$ and call $\tilde{W}$ a generalized Witt modular Lie superalgebra of Cartan type.

Let

$$
G_{i}=\operatorname{span}_{F}\left\{x^{(\alpha)} x^{\mu} y^{\lambda}|| \alpha|+| \mu \mid=i\right\} .
$$

Then $G=\bigoplus_{i=0}^{\xi} G_{i}$ is a $Z$-graded associative superalgebra, where $\xi=\sum_{i=1}^{n} \pi_{i}+q$. Set

$$
\delta\left(j, Y_{2}\right)= \begin{cases}1 & \text { if } j \in Y_{2}, \\ 0 & \text { if } j \notin Y_{2}\end{cases}
$$


Let

$$
\tilde{W}_{i}=\operatorname{span}_{F}\left\{x^{(\alpha)} x^{\mu} y^{\lambda} D_{j}|j \in S,| \alpha|+| \mu \mid+\delta\left(j, Y_{2}\right)-1=i\right\} .
$$

Then $\tilde{W}=\bigoplus_{i=-1}^{\xi} \tilde{W}_{i}$ is a $Z$-graded Lie superalgebra.

Lemma 2.1. Let $f \in G$. If $D_{i}(f)=0$ for all $i \in Y$, then $f \in G_{0}$.

Proof. Without loss of generality, we may assume that $f$ has the form $f=x^{(\alpha)} x^{\mu} y^{\lambda}$. For any $i \in Y_{0} \cup Y_{1}$, our assumption forces $\alpha=0$ and $\mu=\emptyset$. Thus, $f=y^{\lambda} \in G_{0}$.

\section{Structure of $\tilde{W}$}

LemMa 3.1. Let

$$
M_{1}=\left\{x^{\left(k_{i} \varepsilon_{i}\right)} D_{j} \mid i \in Y_{0}, j \in Y, 0 \leq k_{i} \leq \pi_{i}\right\}
$$

let

$$
M_{2}=\left\{x_{l} D_{t} \mid l \in Y_{1}, t \in Y_{2}\right\}
$$

and let

$$
M=M_{1} \cup M_{2} \cup \tilde{W}_{-1} \cup \tilde{W}_{0} .
$$

Then $\tilde{W}$ is generated by the set $M$.

Proof. Let $X$ be the subalgebra of $\tilde{W}$ generated by $M$. We proceed in several steps to show that $\tilde{W}=X$.

Step 1. We show that $x^{\pi} y^{\lambda} D_{1} \in X$. In order to prove the result, we first show that

$$
x^{\left(\pi_{1} \varepsilon_{1}+\cdots+\pi_{t} \varepsilon_{t}\right)} D_{1} \in X
$$

by induction on $t$, where $t \in Y_{0}$.

If $t=1$, then $x^{\left(\pi_{1} \varepsilon_{1}\right)} D_{1} \in M_{1} \subseteq X$. Suppose that $x^{\left(\pi_{1} \varepsilon_{1}+\cdots+\pi_{t-1} \varepsilon_{t-1}\right)} D_{1} \in X$. We can easily verify that

$$
x^{\left(\pi_{t} \varepsilon_{t}\right)} x_{1} D_{1}=\left[x^{\left(\pi_{t} \varepsilon_{t}\right)} D_{1}, x^{\left(2 \varepsilon_{1}\right)} D_{1}\right] \in X .
$$

Moreover, we get

$$
x^{\left(\pi_{1} \varepsilon_{1}+\cdots+\pi_{t} \varepsilon_{t}\right)} D_{1}=1 / 2\left[x^{\left(\pi_{1} \varepsilon_{1}+\cdots+\pi_{t-1} \varepsilon_{t-1}\right)} D_{1}, x^{\left(\pi_{t} \varepsilon_{t}\right)} x_{1} D_{1}\right] \in X .
$$

The induction is completed and $x^{\pi} D_{1} \in X$. Since

$$
x_{1} y^{\lambda} D_{1}=\left[y^{\lambda} D_{1}, x^{\left(2 \varepsilon_{1}\right)} D_{1}\right] \in\left[\tilde{W}_{-1}, M_{1}\right] \subseteq X,
$$

we see that

$$
x^{\pi} y^{\lambda} D_{1}=1 / 2\left[x^{\pi} D_{1}, x_{1} y^{\lambda} D_{1}\right] \in X .
$$

Step 2. We now show that $x^{\pi} x^{E} y^{\lambda} D_{i} \in X$ for $i \in S$. We consider three cases below. 
Case 1. Suppose that $i \in Y_{0}$. We first show that $x_{n+1} \cdots x_{k} D_{1} \in X$ by induction on $k$, where $k \in Y_{1}$. If $k=n+1$, then $x_{n+1} D_{1} \in \tilde{W}_{0} \subseteq X$. Suppose that $x_{n+1} \cdots x_{k-1} D_{1} \in X$. One can easily verify that

$$
x_{1} x_{k} D_{1}=\left[x_{k} D_{1}, x^{\left(2 \varepsilon_{1}\right)} D_{1}\right] \in X .
$$

Moreover,

$$
x_{n+1} \cdots x_{k} D_{1}=\left[x_{n+1} \cdots x_{k-1} D_{1}, x_{1} x_{k} D_{1}\right] \in X .
$$

The induction is completed and $x^{E} D_{1} \in X$.

Since

$$
x^{E} y^{\lambda} D_{1}=\left[x^{E} D_{1}, x_{1} y^{\lambda} D_{1}\right] \in X,
$$

we see that

$$
x_{1} x^{E} y^{\lambda} D_{i}=\left[x^{E} y^{\lambda} D_{1}, x^{\left(2 \varepsilon_{1}\right)} D_{i}\right] \in X
$$

for any $i \in Y_{0}$. Moreover,

$$
x^{\pi} x^{E} y^{\lambda} D_{1}=1 / 2\left[x^{\pi} D_{1}, x_{1} x^{E} y^{\lambda} D_{1}\right] \in X
$$

and

$$
x^{\pi} x^{E} y^{\lambda} D_{i}=\left[x^{\pi} D_{1}, x_{1} x^{E} y^{\lambda} D_{i}\right] \in X
$$

when $i \neq 1$.

Case 2. Suppose that $i \in Y_{1}$. By Case 1, we deduce that

$$
x^{\pi} x^{E} y^{\lambda} D_{i}=\left[x^{\pi} x^{E} y^{\lambda} D_{1}, x_{1} D_{i}\right] \in X .
$$

Case 3. Suppose that $i \in Y_{2}$. Noting that $x_{l} D_{i} \in M_{2}$ for any $l \in Y_{1}$, we deduce from Case 2 that

$$
x^{\pi} x^{E} y^{\lambda} D_{i}=\left[x^{\pi} x^{E} y^{\lambda} D_{l}, x_{l} D_{i}\right] \in X .
$$

Step 3. We shall show that

$$
x^{(\alpha)} x^{\mu} y^{\lambda} D_{i} \in X
$$

for any $i \in S$ by induction on $(|\pi|+|E|)-(|\alpha|+|\mu|)$, which we call $t$.

Let $t=0$. By Step 2, we see that $x^{\pi} x^{E} y^{\lambda} D_{i} \in X$ for any $i \in S$. Let $t \geq 1$. Suppose that the result is true for $t-1$. We consider the two cases $|\alpha|<|\pi|$ and $|\alpha|=|\pi|$ separately.

If $|\alpha|<|\pi|$, then there exists $k \in Y_{0}$ such that $x^{\left(\alpha+\varepsilon_{k}\right)} x^{\mu} y^{\lambda} \in G$. By our inductive hypothesis, $x^{\left(\alpha+\varepsilon_{k}\right)} x^{\mu} y^{\lambda} D_{i} \in X$. Moreover,

$$
x^{(\alpha)} x^{\mu} y^{\lambda} D_{i}=\left[D_{k}, x^{\left(\alpha+\varepsilon_{k}\right)} x^{\mu} y^{\lambda} D_{i}\right] \in X .
$$

If $|\alpha|=|\pi|$, then $|\mu|<|E|$ since $t \geq 1$. Consequently, there exists $k \in Y_{1}$ such that $x_{k} x^{\mu} \neq 0$. By our inductive hypothesis, $x^{(\alpha)} x_{k} x^{\mu} y^{\lambda} D_{i} \in X$. Moreover,

$$
x^{(\alpha)} x^{\mu} y^{\lambda} D_{i}=\left[D_{k}, x^{(\alpha)} x_{k} x^{\mu} y^{\lambda} D_{i}\right] \in X .
$$

Hence, $\tilde{W}=X$ and the proof is completed. 
Lemma 3.2. Let $l=|H|=p^{m}$ and let

$$
\Delta=\left\{\sum_{i=1}^{l} a_{i} y^{\lambda_{i}} \mid \lambda_{i} \in H, a_{i} \in F, \sum_{i=1}^{l} a_{i}=0\right\} .
$$

Then $\Delta$ is an ideal of $Q(m)$ and $Q(m)=\Delta \oplus F 1$.

Proof. Suppose that $f=\sum_{i=1}^{l} a_{i} y^{\lambda_{i}} \in \Delta$ and $g=\sum_{j=1}^{l} b_{j} y^{\lambda_{j}} \in Q(m)$, where $a_{i}, b_{j} \in F$ and $\lambda_{i}, \lambda_{j} \in H$. Then $\sum_{i=1}^{l} a_{i}=0$. Write $h:=f g=\sum_{k=1}^{l} c_{k} y^{\lambda_{k}}$, where $c_{k} \in F, \lambda_{k} \in H$. Then

$$
\left(\sum_{i=1}^{l} a_{i} y^{\lambda_{i}}\right)\left(\sum_{j=1}^{l} b_{j} y^{\lambda_{j}}\right)=\sum_{k=1}^{l} c_{k} y^{\lambda_{k}}
$$

and we conclude that

$$
\sum_{i, j=1}^{l} a_{i} b_{j} y^{\lambda_{i}+\lambda_{j}}=\sum_{k=1}^{l} c_{k} y^{\lambda_{k}}
$$

Since $y^{\lambda_{i}+\lambda_{j}} \neq 0$, we see that

$$
\sum_{k=1}^{l} c_{k}=\sum_{i, j=1}^{l} a_{i} b_{j}=\left(\sum_{i=1}^{l} a_{i}\right)\left(\sum_{j=1}^{l} b_{j}\right)=0 .
$$

Hence, $h \in \Delta$ and $\Delta$ is an ideal of $Q(m)$.

Let $f=\sum_{i=1}^{l} a_{i} y^{\lambda_{i}}$ be any element of $Q(m)$. Then $f-\sum_{i=1}^{l} a_{i} \cdot 1 \in \Delta$ and we conclude that $Q(m)=\Delta+F 1$. Clearly, $\Delta \cap F 1=\{0\}$. Hence, $Q(m)=\Delta \oplus F 1$.

Lemma 3.3. Let

$$
\Gamma=\operatorname{span}_{F}\{g h \mid g \in O(n, t) \otimes \Lambda(q), h \in \Delta\}
$$

and let

$$
\bar{J}=\left\{\sum_{i=1}^{s} f_{i} D_{i} \mid f_{i} \in \Gamma, D_{i_{k}} \cdots D_{i_{2}} D_{i_{1}}\left(f_{i}\right) \in \Gamma \forall i_{k} \in S, 1 \leq k \leq s\right\} .
$$

Then $\Gamma$ is an ideal of $G$ and $\bar{J}$ is an ideal of $\tilde{W}$.

Proof. Let $f \in G$. Without loss of generality, we may suppose that $f=g^{\prime} h^{\prime}$, where $g^{\prime} \in O(n, t) \otimes \Lambda(q)$ and $h^{\prime} \in Q(m)$. Suppose that $g h \in \Gamma$, where $g \in O(n, t) \otimes \Lambda(q)$ and $h \in \Delta$. Then

$$
f(g h)=\left(g^{\prime} h^{\prime}\right)(g h)=\left(g^{\prime} g\right)\left(h^{\prime} h\right) \in \Gamma
$$

by Lemma 3.2. Similarly, $(g h) f \in \Gamma$. Thus, $\Gamma$ is an ideal of $G$.

Now suppose that $A=\sum_{i=1}^{s} g_{i} D_{i} \in \tilde{W}$ and $B=\sum_{j=1}^{s} f_{j} D_{j} \in \bar{J}$, where $g_{i} \in G, f_{j} \in \Gamma$ and $D_{i_{k}} \cdots D_{i_{2}} D_{i_{1}}\left(f_{j}\right) \in \Gamma$. By $(2.1)$, we see that

$$
[A, B]=\sum_{i, j=1}^{s} g_{i} D_{i}\left(f_{j}\right) D_{j}-\sum_{i, j=1}^{s}(-1)^{\left|g_{i} D_{i}\right|\left|f_{j} D_{j}\right|} f_{j} D_{j}\left(g_{i}\right) D_{i} .
$$


By our assumption, $D_{i_{k}} \cdots D_{i_{2}} D_{i_{1}}\left(f_{j}\right) \in \Gamma$. Putting $k=1$, we deduce that $D_{i}\left(f_{j}\right) \in \Gamma$. Consequently, $g_{i} D_{i}\left(f_{j}\right) \in \Gamma$ and $f_{j} D_{j}\left(g_{i}\right) \in \Gamma$. Moreover, we can easily deduce that

$$
D_{i_{k}} \cdots D_{i_{2}} D_{i_{1}}\left(g_{i} D_{i}\left(f_{j}\right)\right) \in \Gamma, \quad D_{i_{k}} \cdots D_{i_{2}} D_{i_{1}}\left(f_{j} D_{j}\left(g_{i}\right)\right) \in \Gamma
$$

by induction on $k$. Hence, $\bar{J}$ is an ideal of $\tilde{W}$.

Suppose that

$$
\bar{X}=\left\{\sum_{i=1}^{s} g_{i} D_{i} \mid g_{i} \in G, \exists k \in\{1, \ldots, s\} \text { such that } i_{k} \in S \text { and } D_{i_{k}} \cdots D_{i_{2}} D_{i_{1}}\left(g_{i}\right) \notin \Gamma\right\} \text {. }
$$

It may be verified that $\bar{X}$ is a subalgebra of $\tilde{W}$. In particular,

$$
\left\{\sum_{i=1}^{s} g_{i} D_{i} \mid g_{i} \in O(n, t) \otimes \Lambda(q)\right\}
$$

is a subalgebra of $\bar{X}$.

Theorem 3.4. The algebra $\tilde{W}$ is an extension of a subalgebra $\bar{X}$ by the ideal $\bar{J}$.

Proof. Let $\sum_{i=1}^{s} f_{i} D_{i}$ be any element of $\tilde{W}$, where $f_{i} \in G$. Without loss of generality, we may suppose that $f_{i}=g_{i} h_{i}$, where $g_{i} \in O(n, t) \otimes \Lambda(q)$ and $h_{i} \in Q(m)$. It follows by Lemma 3.2 that

$$
f_{i}=g_{i}\left(h_{i}^{\prime}+a_{i} 1\right)=g_{i} h_{i}^{\prime}+a_{i} g_{i},
$$

where $h_{i}^{\prime} \in \Delta, a_{i} \in F$. Thus,

$$
\begin{aligned}
& \sum_{i=1}^{s} f_{i} D_{i}=\sum_{i=1}^{s}\left(g_{i} h_{i}^{\prime}\right) D_{i}+\sum_{i=1}^{s}\left(a_{i} g_{i}\right) D_{i} \\
& =\sum_{D_{i_{k}} \cdots D_{i_{2}} D_{i_{1}}\left(g_{i} h_{i}^{\prime}\right) \in \Gamma}\left(g_{i} h_{i}^{\prime}\right) D_{i}+\sum_{D_{i_{k}} \cdots D_{i_{2}} D_{i_{1}}\left(g_{i} h_{i}^{\prime}\right) \notin \Gamma}\left(g_{i} h_{i}^{\prime}\right) D_{i}+\sum_{i=1}^{s}\left(a_{i} g_{i}\right) D_{i} \\
& \in \bar{J}+\bar{X} \text {. }
\end{aligned}
$$

It is clear that $\bar{X} \cap \bar{J}=\{0\}$. By Lemma 3.3, $\tilde{W}$ is an extension of $\bar{X}$ by $\bar{J}$.

\section{Derivation superalgebra of $\tilde{W}$}

Let $L=\bigoplus_{i \in Z} L_{i}$ be a $Z$-graded superalgebra. Let $x \in L$. If there exists $i \in Z$ such that $x \in L_{i}$, then we call $x$ a $Z$-homogeneous element and $i$ the $Z$-degree of $x$. As usual, the derivation superalgebra of $\tilde{W}$ is a $Z$-graded Lie superalgebra, that is, $\operatorname{der} \tilde{W}=\bigoplus_{t \in J} \operatorname{der}_{t} \tilde{W}$, where

$$
\operatorname{der}_{t} \tilde{W}:=\left\{\varphi \in \operatorname{der} \tilde{W} \mid \varphi\left(\tilde{W}_{i}\right) \subset \tilde{W}_{t+i}\right\}, \quad J=\{-\xi-1,-\xi, \ldots, \xi, \xi+1\} .
$$


Set

$$
\tau(i)= \begin{cases}\pi_{i} & \text { if } i \in Y_{0}, \\ 1 & \text { if } i \in Y_{1} .\end{cases}
$$

Define a linear mapping $\rho_{i}: G \rightarrow G$ such that

$$
\rho_{i}\left(x^{(\alpha)} x^{\mu} y^{\lambda}\right)= \begin{cases}x^{\left(\alpha+\varepsilon_{i}\right)} x^{\mu} y^{\lambda} & \text { if } i \in Y_{0} \text { and } \alpha+\varepsilon_{i} \in A(\boldsymbol{t}), \\ x^{(\alpha)} x_{i} x^{\mu} y^{\lambda} & \text { if } i \in Y_{1}, \\ \lambda_{i}^{-1} x^{(\alpha)} x^{\mu} y^{\lambda} & \text { if } i \in Y_{2} \text { and } \lambda_{i} \neq 0 .\end{cases}
$$

We use the convention that $\rho_{i}\left(x^{(\alpha)} x^{\mu} y^{\lambda}\right)=0$ for $\alpha+\varepsilon_{i} \notin A(t)$ or $\lambda_{i}=0$.

Definition 4.1. An element $f$ of $G$ is said to be of $D_{i}$-type if $D_{i}^{\tau(i)}(f)=0$ for $i \in Y$ and $D_{i}^{p-1}(f)=f$ for $i \in Y_{2}$.

\section{Lemma 4.2. Suppose that $f \in G$.}

(i) If $i \in Y_{2}$, then $f$ is of $D_{i}$-type if and only if $\lambda_{i}^{p-1}=1$.

(ii) $D_{i}(f)$ is of $D_{i}$-type for any $i \in S$.

Proof. Part (i) is obvious.

We now consider part (ii). For $i \in Y$, it is clear that

$$
D_{i}^{\tau(i)}\left(D_{i}(f)\right)=D_{i}^{\tau(i)+1}(f)=0 .
$$

For $i \in Y_{2}$, we may assume that $f=x^{(\alpha)} x^{\mu} y^{\lambda}$. Since $\lambda_{i}^{p}=\lambda_{i}$, we see that

$$
D_{i}^{p-1}\left(D_{i}(f)\right)=D_{i}^{p}(f)=\lambda_{i}^{p} x^{(\alpha)} x^{\mu} y^{\lambda}=\lambda_{i} x^{(\alpha)} x^{\mu} y^{\lambda}=D_{i}(f) .
$$

Hence, $D_{i}(f)$ is of $D_{i}$-type.

\section{Lemma 4.3. Suppose that $i, j \in S$ and $i \neq j$. Then:}

(i) if $f \in G$ is of $D_{i}$-type, then $D_{i} \rho_{i}(f)=f$;

(ii) we have the equality

$$
D_{i} \rho_{j}=(-1)^{\tilde{i} \tilde{\rho}} \rho_{j} D_{i} .
$$

Proof. To prove (i), suppose that $i \in Y_{2}$ and $f=x^{(\alpha)} x^{\mu} y^{\lambda}$. Since $f$ is of $D_{i}$-type, we deduce from Lemma 4 .2(i) that $\lambda_{i} \neq 0$. Thus,

$$
D_{i} \rho_{i}(f)=D_{i}\left(\lambda_{i}^{-1} x^{(\alpha)} x^{\mu} y^{\lambda}\right)=x^{(\alpha)} x^{\mu} y^{\lambda}=f .
$$

The remaining cases where $i \in Y_{0} \cup Y_{1}$ are similar.

Part (ii) is obvious.

Lemma 4.4. Let $f_{t_{1}}, f_{t_{2}}, \ldots, f_{t_{k}} \in G$, where $t_{1}, t_{2}, \ldots, t_{k} \in S$. If $f_{t_{1}}, f_{t_{2}}, \ldots, f_{t_{k}}$ are of $D_{i}$-type and $D_{i}\left(f_{j}\right)=(-1)^{\tilde{\imath} \tilde{J}} D_{j}\left(f_{i}\right)$ for any $i, j \in\left\{t_{1}, t_{2}, \ldots, t_{k}\right\}$, there exists $f \in G$ such that $D_{i}(f)=f_{i}$ for all $i=t_{1}, t_{2}, \ldots, t_{k}$. 
Proof. We use induction on $k$. Let $k=1$ and $f=\rho_{t_{1}}\left(f_{t_{1}}\right)$. It follows from Lemma 4.3(i) that $D_{t_{1}}(f)=D_{t_{1}} \rho_{t_{1}}\left(f_{t_{1}}\right)=f_{t_{1}}$.

Suppose that there exists $g \in G$ such that $D_{i}(g)=f_{i}$ whenever $i=t_{1}, t_{2}, \ldots, t_{k-1}$. Let $f=g+\rho_{t_{k}}\left(f_{t_{k}}-D_{t_{k}}(g)\right)$. By our inductive hypothesis and Lemma 4.3(ii), we deduce that

$$
\begin{aligned}
D_{i}(f) & =D_{i}(g)+D_{i} \rho_{t_{k}}\left(f_{t_{k}}-D_{t_{k}}(g)\right) \\
& =f_{i}+(-1)^{\tilde{t}_{k} \tilde{\imath}} \rho_{t_{k}}\left(D_{i}\left(f_{t_{k}}\right)-D_{i} D_{t_{k}}(g)\right) \\
& =f_{i}+(-1)^{\tilde{t}_{k} \tilde{l}} \rho_{t_{k}}\left((-1)^{\tilde{t}_{k} \tilde{l}} D_{t_{k}}\left(f_{i}\right)-(-1)^{\tilde{t}_{k} \tilde{l}} D_{t_{k}} D_{i}(g)\right) \\
& =f_{i} .
\end{aligned}
$$

We have to show that $D_{t_{k}}(f)=f_{t_{k}}$. By Lemma 4.2(ii), $D_{t_{k}}(g)$ is of $D_{t_{k}}$-type. Consequently, $f_{t_{k}}-D_{t_{k}}(g)$ is also of $D_{t_{k}}$-type. By Lemma 4.3(i),

$$
D_{t_{k}}(f)=D_{t_{k}}(g)+D_{t_{k}} \rho_{t_{k}}\left(f_{t_{k}}-D_{t_{k}}(g)\right)=D_{t_{k}}(g)+\left(f_{t_{k}}-D_{t_{k}}(g)\right)=f_{t_{k}}
$$

and our result follows.

Lemma 4.5. We have $C(\tilde{W})=0$, where $C(\tilde{W})$ denotes the center of $\tilde{W}$.

Proof. Let $D \in C(\tilde{W})$ and write $D=\sum_{k=1}^{s} f_{k} D_{k}$, where $f_{k} \in G$. For any $i \in S$,

$$
\left[D, D_{i}\right]=\left[\sum_{k=1}^{s} f_{k} D_{k}, D_{i}\right]=-(-1)^{\left|f_{k} D_{k}\right| \tilde{\imath}} \sum_{k=1}^{s} D_{i}\left(f_{k}\right) D_{k}=0 .
$$

This implies that $D_{i}\left(f_{k}\right)=0$ for all $i \in S$.

Moreover, by Lemma 2.1, we see that $f_{k} \in G_{0}$ for all $k \in S$. For $j \in Y$ and $t \in Y_{2}, m \in$ $Y_{0}$, one calculates

$$
\left[D, x_{j} D_{j}+y_{t} D_{m}\right]=\left[\sum_{k=1}^{s} f_{k} D_{k}, x_{j} D_{j}+y_{t} D_{m}\right]=f_{j} D_{j}+f_{t} y_{t} D_{m}=0 .
$$

It follows that $f_{j}=f_{t}=0$ and $D=0$.

Lemma 4.6. Let $L$ be a centerless Lie superalgebra. Let $\varphi \in \mathrm{h}(\operatorname{der} L), x \in L_{\overline{0}}$ and $x_{1} \in L$. If there exists $k \geq 1$ such that $(\operatorname{ad} x)^{p^{k}}=\operatorname{ad} x_{1}$, then $\varphi\left(x_{1}\right)=(\operatorname{ad} x)^{p^{k}-1} \varphi(x)$.

Proof. The proof is similar to that of [11, Lemma 8.1, p. 191].

Lemma 4.7. Let $\varphi \in \mathrm{h}\left(\operatorname{der}_{t} \tilde{W}\right)$, where $t \in J$ and $t \geq 0$. Then there exists $A \in \tilde{W}_{t}$ such that $\varphi\left(D_{i}\right)=\operatorname{ad} A\left(D_{i}\right)$ for all $i \in S$.

Proof. Let $\varphi\left(D_{i}\right)=\sum_{k=1}^{s} f_{k i} D_{k}$, where $f_{k i} \in G$. This implies that $|\varphi|+\tilde{l}=\left|f_{k i}\right|+\tilde{k}$. Since $\left[D_{i}, D_{j}\right]=0$ for any $j \in S$, we see that

$$
\left[\sum_{k=1}^{s} f_{k i} D_{k}, D_{j}\right]+(-1)^{|\varphi| \hat{\imath}}\left[D_{i}, \sum_{k=1}^{s} f_{k j} D_{k}\right]=0
$$


It follows that

$$
\sum_{k=1}^{s}\left[(-1)^{|\varphi| \tilde{l}} D_{i}\left(f_{k j}\right)-(-1)^{\left(\left|f_{k i}\right|+\tilde{k}\right) \tilde{J}} D_{j}\left(f_{k i}\right)\right] D_{k}=0 .
$$

Since $|\varphi|+\tilde{l}=\left|f_{k i}\right|+\tilde{k}$, we see that

$$
D_{i}\left((-1)^{|\varphi| \tilde{\jmath}} f_{k j}\right)=(-1)^{\tilde{\imath} \tilde{\jmath}} D_{j}\left((-1)^{|\varphi| \tilde{\imath}} f_{k i}\right) .
$$

For our purposes, it is enough to suppose that $f_{k i}$ is of $D_{i}$-type. We treat the three possible cases separately.

Case 1. Suppose that $i \in Y_{0}$. Since $\left(\operatorname{ad} D_{i}\right)^{\pi_{i}+1}=0$, we deduce from Lemma 4.6 that $\left(\operatorname{ad} D_{i}\right)^{\pi_{i}}\left(\varphi\left(D_{i}\right)\right)=0$. This implies that $\left(\operatorname{ad} D_{i}\right)^{\pi_{i}}\left(\sum_{k=1}^{s} f_{k i} D_{k}\right)=0$. It follows that $D_{i}^{\pi_{i}}\left(f_{k i}\right)=0$.

Case 2. Suppose that $i \in Y_{1}$. Putting $j=i$ in (4.1) enables us to deduce that $D_{i}\left(f_{k i}\right)=0$.

Case 3. Suppose that $i \in Y_{2}$. Since $\lambda_{i}^{p}=\lambda_{i}$, we see that

$$
\left(\operatorname{ad} D_{i}\right)^{p}\left(x^{(\alpha)} x^{u} y^{\lambda} D_{j}\right)=\lambda_{i}^{p} x^{(\alpha)} x^{u} y^{\lambda} D_{j}=\lambda_{i} x^{(\alpha)} x^{u} y^{\lambda} D_{j}=\operatorname{ad} D_{i}\left(x^{(\alpha)} x^{u} y^{\lambda} D_{j}\right) .
$$

It follows that

$$
\left(\operatorname{ad} D_{i}\right)^{p-1}\left(\varphi\left(D_{i}\right)\right)=\varphi\left(D_{i}\right)
$$

by Lemma 4.6. Consequently,

$$
\left(\operatorname{ad} D_{i}\right)^{p-1}\left(\sum_{k=1}^{s} f_{k i} D_{k}\right)=\sum_{k=1}^{s} D_{i}^{p-1}\left(f_{k i}\right) D_{k}=\sum_{k=1}^{s} f_{k i} D_{k}
$$

This implies that $D_{i}^{p-1}\left(f_{k i}\right)=f_{k i}$. Hence, $f_{k i}$ is of $D_{i}$-type for all $k, i \in S$.

Equation (4.1) shows that $\left\{(-1)^{|\varphi| \tau} f_{k i} \mid i \in S\right\}$ satisfies the conditions of Lemma 4.4. Thus, there exists $g_{k} \in G$ such that $D_{i}\left(g_{k}\right)=(-1)^{|\varphi| \imath} f_{k i}$. This implies that $\tilde{\imath}+\left|g_{k}\right|=\left|f_{k i}\right|$. Note that $|\varphi|=\left|g_{k}\right|+\tilde{k}$. Write

$$
B:=-\sum_{k=1}^{s} g_{k} D_{k} \in \tilde{W}
$$

One deduces that

$$
\left[B, D_{i}\right]=\sum_{k=1}^{s}(-1)^{\left(\left|g_{k}\right|+\tilde{k}\right) \tilde{l}} D_{i}\left(g_{k}\right) D_{k}=\sum_{k=1}^{s}(-1)^{|\varphi| \tilde{\imath}} D_{i}\left(g_{k}\right) D_{k}=\sum_{k=1}^{s} f_{k i} D_{k}=\varphi\left(D_{i}\right) .
$$

Since $\tilde{W}$ is $Z$-graded, we may suppose that $B=\sum_{l=-1}^{\xi} B_{l}$, where $B_{l} \in \tilde{W}_{l}$. It follows that $\varphi\left(D_{i}\right)=\left[B_{t}, D_{i}\right]$. Thereby, we find $A=B_{t} \in \tilde{W}_{t}$ such that $\varphi\left(D_{i}\right)=\operatorname{ad} A\left(D_{i}\right)$ for $i \in S$. 
Write

$$
\Theta:=Q(m)^{m_{2}}=Q(m) \times Q(m) \times \cdots \times Q(m) .
$$

For

$$
\theta=\left(h_{s+1}(y), h_{s+2}(y), \ldots, h_{s_{1}}(y)\right) \in \Theta
$$

define

$$
\tilde{\theta}: H \longrightarrow Q(m), \quad \lambda \longmapsto \sum_{j=s+1}^{s_{1}} \lambda_{j} h_{j}(y) .
$$

For $\lambda, \eta \in H$, we are able to verify that

$$
\tilde{\theta}(\lambda+\eta)=\tilde{\theta}(\lambda)+\tilde{\theta}(\eta) .
$$

For $\theta \in \Theta$, define a linear mapping $D_{\theta}: \tilde{W} \longrightarrow \tilde{W}$ such that

$$
D_{\theta}\left(x^{(\alpha)} x^{\mu} y^{\lambda} D_{i}\right)=\tilde{\theta}(\lambda) x^{(\alpha)} x^{\mu} y^{\lambda} D_{i} \quad \forall i \in S .
$$

Lemma 4.8. For any $\theta \in \Theta$, we have $D_{\theta} \in \operatorname{der}_{\overline{0}}(\tilde{W})$.

Proof. For $i \in Y_{0}$ and $k \in Y_{2}$, a direct computation shows that

$$
\left[x^{(\alpha)} x^{\mu} y^{\lambda} D_{i}, x^{(\beta)} x^{\nu} y^{\eta} D_{k}\right]=x^{(\alpha)} x^{\mu} x^{\nu} y^{\eta}\left[y^{\lambda} D_{i}, x^{(\beta)} D_{k}\right] .
$$

Consequently,

$$
\begin{aligned}
D_{\theta}\left[x^{(\alpha)}\right. & \left.x^{\mu} y^{\lambda} D_{i}, x^{(\beta)} x^{v} y^{\eta} D_{k}\right] \\
& =D_{\theta}\left(x^{(\alpha)} x^{\mu} x^{v} y^{\eta} y^{\lambda} D_{i}\left(x^{(\beta)}\right) D_{k}-x^{(\alpha)} x^{\mu} x^{v} y^{\eta} x^{(\beta)} D_{k}\left(y^{\lambda}\right) D_{i}\right) \\
& =D_{\theta}\left(x^{(\alpha)} x^{\mu} x^{v} y^{\lambda+\eta} D_{i}\left(x^{(\beta)}\right) D_{k}\right)-D_{\theta}\left(\lambda_{k} x^{(\alpha)} x^{(\beta)} x^{\mu} x^{v} y^{\lambda+\eta} D_{i}\right) \\
& =\tilde{\theta}(\lambda+\eta) x^{(\alpha)} x^{\mu} x^{v} y^{\eta}\left(y^{\lambda} D_{i}\left(x^{(\beta)}\right) D_{k}-x^{(\beta)} D_{k}\left(y^{\lambda}\right) D_{i}\right) \\
& =(\tilde{\theta}(\lambda)+\tilde{\theta}(\eta)) x^{(\alpha)} x^{\mu} x^{v} y^{\eta}\left[y^{\lambda} D_{i}, x^{(\beta)} D_{k}\right] \\
& =(\tilde{\theta}(\lambda)+\tilde{\theta}(\eta))\left[x^{(\alpha)} x^{\mu} y^{\lambda} D_{i}, x^{(\beta)} x^{v} y^{\eta} D_{k}\right] \\
& =\left[D_{\theta}\left(x^{(\alpha)} x^{\mu} y^{\lambda} D_{i}\right), x^{(\beta)} x^{v} y^{\eta} D_{k}\right]+\left[x^{(\alpha)} x^{\mu} y^{\lambda} D_{i}, D_{\theta}\left(x^{(\beta)} x^{v} y^{\eta} D_{k}\right)\right] .
\end{aligned}
$$

Hence, we conclude that $D_{\theta} \in \operatorname{der}_{\overline{0}}(\tilde{W})$. The argument for the remaining cases is similar.

Lemma 4.9. Let $\varphi \in \mathrm{h}(\operatorname{der} \tilde{W})$. If $\varphi\left(D_{j}\right)=0$ for all $j \in S$, then there exists $\theta \in \Theta$ such that $\varphi\left(y^{\lambda} D_{i}\right)=D_{\theta}\left(y^{\lambda} D_{i}\right)$ for any $\lambda \in H$ and $i \in Y$.

Proof. We proceed in several steps.

Step 1. Let $\varphi\left(y^{\lambda} D_{i}\right)=\sum_{k=1}^{s} g_{k i \lambda} D_{k}$, where $g_{k i \lambda} \in G$. Since $\left[D_{j}, y^{\lambda} D_{i}\right]=0$ for $j \in Y$, we see that

$$
\left[\varphi\left(D_{j}\right), y^{\lambda} D_{i}\right]+(-1)^{|\varphi| \tilde{J}}\left[D_{j}, \varphi\left(y^{\lambda} D_{i}\right)\right]=0 .
$$

Consequently, it follows by our assumption that $\varphi\left(D_{j}\right)=0$ that

$$
\left[D_{j}, \varphi\left(y^{\lambda} D_{i}\right)\right]=\left[D_{j}, \sum_{k=1}^{s} g_{k i \lambda} D_{k}\right]=\sum_{k=1}^{s} D_{j}\left(g_{k i \lambda}\right) D_{k}=0 .
$$

We now deduce from Lemma 2.1 that $g_{k i \lambda} \in G_{0}$ for all $k \in S$. 
Step 2. Let $\varphi\left(x_{i} D_{i}\right)=\sum_{k=1}^{s} a_{k} D_{k}$, where $a_{k} \in G$. Since $\left[D_{i}, x_{i} D_{i}\right]=D_{i}$, we see that

$$
\left[D_{i}, \sum_{k=1}^{s} a_{k} D_{k}\right]=\sum_{k=1}^{s} D_{i}\left(a_{k}\right) D_{k}=0
$$

This means that $a_{k} \in G_{0}$ by Lemma 2.1.

Since $\left[y^{\lambda} D_{i}, x_{i} D_{i}\right]=y^{\lambda} D_{i}$, we deduce that

$$
\left[\sum_{k=1}^{s} g_{k i \lambda} D_{k}, x_{i} D_{i}\right]+(-1)^{|\varphi| \hat{i}}\left[y^{\lambda} D_{i}, \sum_{k=1}^{s} a_{k} D_{k}\right]=\sum_{k=1}^{s} g_{k i \lambda} D_{k} .
$$

This implies that

$$
g_{i i \lambda} D_{i}-\sum_{k \in Y_{2}}(-1)^{|\varphi| \tilde{\imath}} a_{k} D_{k}\left(y^{\lambda}\right) D_{i}=\sum_{k=1}^{s} g_{k i \lambda} D_{k}
$$

It follows that $g_{k i \lambda}=0$ for all $k \in S \backslash\{i\}$ and $\varphi\left(y^{\lambda} D_{i}\right)=g_{i i \lambda} D_{i}$. We abbreviate $g_{i i \lambda}$ to $g_{i \lambda}$. Set $h_{i \lambda}(y)=g_{i \lambda} y^{-\lambda}$. Then

$$
\varphi\left(y^{\lambda} D_{i}\right)=g_{i \lambda} D_{i}=h_{i \lambda}(y) y^{\lambda} D_{i} .
$$

Step 3. We claim that

$$
h_{i \lambda}(y)+h_{j \eta}(y)=h_{j(\lambda+\eta)}(y)
$$

for any $\lambda, \eta \in H$ and $i, j \in Y$.

Suppose that $\varphi\left(x_{i} y^{\eta} D_{j}\right)=\sum_{k=1}^{s} f_{k} D_{k}$, where $f_{k} \in G$. Since $\left[D_{i}, x_{i} y^{\eta} D_{j}\right]=y^{\eta} D_{j}$, we deduce that

$$
(-1)^{|\varphi| \tilde{i}}\left[D_{i}, \sum_{k=1}^{s} f_{k} D_{k}\right]=(-1)^{|\varphi| \tilde{\imath}} \sum_{k=1}^{s} D_{i}\left(f_{k}\right) D_{k}=h_{j \eta}(y) y^{\eta} D_{j}
$$

This implies that $D_{i}\left(f_{k}\right)=0$ for all $k \in S \backslash\{j\}$ and $D_{i}\left(f_{j}\right)=(-1)^{|\varphi| \tau} h_{j \eta}(y) y^{\eta}$. Therefore, we may assume that $f_{j}=(-1)^{|\varphi| \tau} h_{j \eta}(y) y^{\eta} x_{i}+g_{j}$, where $g_{j} \in G$ and $D_{i}\left(g_{j}\right)=0$. Since $\left[y^{\lambda} D_{i}, x_{i} y^{\eta} D_{j}\right]=y^{\lambda+\eta} D_{j}$, we deduce that

$$
\begin{aligned}
& {\left[h_{i \lambda}(y) y^{\lambda} D_{i}, x_{i} y^{\eta} D_{j}\right]+(-1)^{|\varphi| \tilde{\imath}}\left[y^{\lambda} D_{i}, \sum_{k=1}^{s} f_{k} D_{k}\right]} \\
& \quad=\left[h_{i \lambda}(y) y^{\lambda} D_{i}, x_{i} y^{\eta} D_{j}\right]+(-1)^{|\varphi| \tilde{\imath}}\left[y^{\lambda} D_{i},(-1)^{|\varphi| \tilde{\imath}} h_{j \eta}(y) y^{\eta} x_{i} D_{j}+g_{j} D_{j}+\sum_{k \neq j} f_{k} D_{k}\right] \\
& \quad=h_{i \lambda}(y) y^{\lambda+\eta} D_{j}+h_{j \eta}(y) y^{\lambda+\eta} D_{j}-\sum_{k \in Y_{2}}(-1)^{\left(|\varphi|+\left|f_{k} D_{k}\right|\right) \tilde{\imath}} f_{k} D_{k}\left(y^{\lambda}\right) D_{i} \\
& =h_{j(\lambda+\eta)}(y) y^{(\lambda+\eta)} D_{j} .
\end{aligned}
$$


In the following, we consider the two cases where $i \neq j$ and $i=j$ separately. If $i \neq j$, then the assertion is obvious. Moreover, we deduce that

$$
\sum_{k \in Y_{2}}(-1)^{\left(|\varphi|+\left|f_{k} D_{k}\right|\right) \tilde{\imath}} f_{k} D_{k}\left(y^{\lambda}\right)=0 .
$$

Hence, if $i=j$, then the equality $h_{i \lambda}(y)+h_{j \eta}(y)=h_{j(\lambda+\eta)}(y)$ also holds. We have established our claim.

Step 4. Since $\lambda, \eta, i, j$ have been chosen randomly,

$$
h_{i \lambda}(y)+h_{j \lambda}(y)=h_{j(2 \lambda)}(y)=h_{j \lambda}(y)+h_{j \lambda}(y) .
$$

We deduce that $h_{i \lambda}(y)=h_{j \lambda}(y)$. We write $h_{i \lambda}(y)$ for $h_{\lambda}(y)$ for any $i \in Y$. Then $\varphi\left(y^{\lambda} D_{i}\right)=$ $h_{\lambda}(y) y^{\lambda} D_{i}$. By Step $3, h_{\lambda}(y)+h_{\eta}(y)=h_{\lambda+\eta}(y)$. In particular,

$$
h_{\bar{\varepsilon}_{k}}(y)+h_{\bar{\varepsilon}_{k}}(y)=h_{2 \bar{\varepsilon}_{k}}(y)=2 h_{\bar{\varepsilon}_{k}}(y), h_{2 \bar{\varepsilon}_{k}}(y)+h_{\bar{\varepsilon}_{k}}(y)=h_{3 \bar{\varepsilon}_{k}}(y)=3 h_{\bar{\varepsilon}_{k}}(y) .
$$

Moreover, we see that $h_{c \bar{\varepsilon}_{k}}(y)=c h_{\bar{\varepsilon}_{k}}(y)$ for any $c \in \Pi$ and $k=r+1, \ldots, s_{1}$. We abbreviate $h_{\bar{\varepsilon}_{k}}(y)$ by $h_{k}(y)$.

Step 5. We now complete the proof. Set

$$
H_{1}=\left\{\lambda \in H \mid \lambda_{k}=0 \forall k=s+1, s+2, \ldots, s_{1}\right\}
$$

and

$$
H_{2}=\left\{\lambda \in H \mid \lambda_{k}=0 \forall k \in Y_{2}\right\} .
$$

For any $\lambda \in H$, we can find $\lambda^{\prime} \in H_{1}$ and $\lambda^{\prime \prime} \in H_{2}$ such that $\lambda=\lambda^{\prime}+\lambda^{\prime \prime}$.

Suppose that $\lambda_{t}$ is the first number of $\lambda^{\prime \prime}$ which is not equal to 0 , where $t$ is one of $s+1, \ldots, s_{1}$. Then

$$
\begin{aligned}
h_{\lambda}(y) & =h_{\lambda^{\prime}+\lambda^{\prime \prime}}(y)=h_{\lambda^{\prime}}(y)+h_{\lambda^{\prime \prime}}(y) \\
& =h_{\lambda^{\prime}}(y)+h_{\lambda_{t} \bar{\varepsilon}_{t}+\cdots+\lambda_{s_{1}} \bar{\varepsilon}_{s_{1}}}(y) \\
& =h_{\lambda^{\prime}}(y)+\lambda_{t} h_{t}(y)+\cdots+\lambda_{s_{1}} h_{s_{1}}(y) \\
& =\lambda_{s+1} h_{s+1}(y)+\cdots+\lambda_{t}\left(\lambda_{t}^{-1} h_{\lambda^{\prime}}(y)+h_{t}(y)\right)+\cdots+\lambda_{s_{1}} h_{s_{1}}(y) .
\end{aligned}
$$

Set

$$
\theta=\left(h_{s+1}(y), \ldots, \lambda_{t}^{-1} h_{\lambda^{\prime}}(y)+h_{t}(y), h_{t+1}(y), \ldots, h_{s_{1}}(y)\right) .
$$

Then $\theta \in \Theta$ and

$$
\varphi\left(y^{\lambda} D_{i}\right)=h_{\lambda}(y) y^{\lambda} D_{i}=\tilde{\theta}(\lambda) y^{\lambda} D_{i}=D_{\theta}\left(y^{\lambda} D_{i}\right) .
$$

This completes the proof.

Lemma 4.10. Let $A \in \tilde{W}$. If $\left[D_{i}, A\right]=\left[y_{j} D_{t}, A\right]=0$ for all $i \in Y, t \in Y_{1}$ and $j \in Y_{2}$, then $A \in \tilde{W}_{-1}$. 
Proof. Suppose that $A=\sum_{k=1}^{s} f_{k} D_{k}$, where $f_{k} \in G$. Then

$$
\left[D_{i}, A\right]=\left[D_{i}, \sum_{k=1}^{s} f_{k} D_{k}\right]=\sum_{k=1}^{s} D_{i}\left(f_{k}\right) D_{k}=0
$$

and we conclude that $D_{i}\left(f_{k}\right)=0$. By Lemma 2.1, this shows that $f_{k} \in G_{0}$ for all $k \in S$. Since $\left[y_{j} D_{t}, A\right]=\left[y_{j} D_{t}, \sum_{k=1}^{s} f_{k} D_{k}\right]=0$, it follows that $f_{j} y_{j} D_{t}=0$. This shows that $f_{j}=0$ for all $j \in Y_{2}$, whence $A=\sum_{k=1}^{r} f_{k} D_{k} \in \tilde{W}_{-1}$.

Lemma 4.11. Let $\varphi \in \mathrm{h}\left(\operatorname{der}_{t} \tilde{W}\right)$, where $t \in J$. Suppose that $k \geq-1$ and $\varphi\left(\tilde{W}_{j}\right)=0$, where $j=-1,0, \ldots, k$. If $k+t \geq-1$, then $\varphi=0$.

Proof. We let $l \geq k$ and show that $\varphi\left(\tilde{W}_{l}\right)=0$ by induction on $l$. By our assumption that $\varphi\left(\tilde{W}_{j}\right)=0$, it will then follow that $\varphi\left(\tilde{W}_{k}\right)=0$.

Suppose that $l>k$ and $\varphi\left(\tilde{W}_{l-1}\right)=0$. Lemma 4.10 allows us to deduce that

$$
\varphi(A) \in \tilde{W}_{-1} \cap \tilde{W}_{l+t}=0,
$$

since $\left[D_{i}, A\right] \in \tilde{W}_{l-1}$ for any $A \in \tilde{W}_{l}$ and $i \in Y$ and $\left[y_{h} D_{v}, A\right] \in \tilde{W}_{l-1}$ for any $h \in Y_{2}$ and $v \in Y_{1}$, while $\varphi\left(D_{i}\right)=\varphi\left(y_{h} D_{v}\right)=0$. Hence, $\varphi\left(\tilde{W}_{l}\right)=0$ and we may conclude that $\varphi=0$.

Proposition 4.12. Let $\varphi \in \mathrm{h}\left(\operatorname{der}_{t} \tilde{W}\right)$, where $t \in J$ and $t \geq 0$. Then there exist $A \in \tilde{W}_{t}$ and $\theta \in \Theta$ such that $\varphi=\operatorname{ad} A+D_{\theta}$.

Proof. By Lemma 4.7, there exists $A \in \tilde{W}_{t}$ such that $\varphi\left(D_{i}\right)=\operatorname{ad} A\left(D_{i}\right)$ for all $i \in S$. Thus, we may find $\theta \in \Theta$ such that $\left(\varphi-\operatorname{ad} A-D_{\theta}\right)\left(y^{\lambda} D_{j}\right)=0$ for any $\lambda \in H$ and $j \in Y$ by Lemma 4.9. This allows us to deduce that $\left(\varphi-\operatorname{ad} A-D_{\theta}\right)\left(\tilde{W}_{-1}\right)=0$ and $\varphi=\operatorname{ad} A+D_{\theta}$ by Lemma 4.11 .

REMARK 4.13. It is possible to add the following conclusions to Proposition 4.12. If $\varphi \in\left(\operatorname{der}_{0} \tilde{W}\right)_{\overline{0}}$, then there exist $A \in \tilde{W}_{t}$ and $\theta \in \Theta$ such that $\varphi=\operatorname{ad} A+D_{\theta}$. Otherwise there exists $A \in \tilde{W}_{t}$ such that $\varphi=\operatorname{ad} A$.

Proposition 4.14. Let $\Omega=\left\{D_{\theta} \mid \theta \in \Theta\right\}$. Then the following statements hold.

(i) The space $\Omega$ is a subspace of $\operatorname{der} \tilde{W}$.

(ii) The intersection ad $\tilde{W} \cap \Omega=\{0\}$.

Proof. We first prove (i). Since $Q(m)$ is a linear space over $F$, we see that $\Theta=Q(m)^{m_{2}}$ is also a linear space over $F$. Suppose that

$$
\theta=\left(h_{s+1}(y), \ldots, h_{s_{1}}(y)\right), \quad \eta=\left(g_{s+1}(y), \ldots, g_{s_{1}}(y)\right)
$$

for any $\theta, \eta \in \Theta$. Then

$$
\theta+\eta=\left(h_{s+1}(y)+g_{s+1}(y), \ldots, h_{s_{1}}(y)+g_{s_{1}}(y)\right) .
$$


For $\lambda \in H$,

$$
\begin{aligned}
\tilde{\theta}(\lambda)+\tilde{\eta}(\lambda) & =\sum_{j=s+1}^{s_{1}} \lambda_{j} h_{j}(y)+\sum_{j=s+1}^{s_{1}} \lambda_{j} g_{j}(y) \\
& =\sum_{j=s+1}^{s_{1}} \lambda_{j}\left(h_{j}(y)+g_{j}(y)\right)=(\theta+\eta)^{\sim}(\lambda) .
\end{aligned}
$$

We deduce that

$$
\begin{aligned}
\left(D_{\theta}+D_{\eta}\right)\left(x^{(\alpha)} x^{\mu} y^{\lambda} D_{i}\right) & =\tilde{\theta}(\lambda) x^{(\alpha)} x^{\mu} y^{\lambda} D_{i}+\tilde{\eta}(\lambda) x^{(\alpha)} x^{\mu} y^{\lambda} D_{i} \\
& =(\theta+\eta)^{\sim}(\lambda) x^{(\alpha)} x^{\mu} y^{\lambda} D_{i} \\
& =D_{\theta+\eta}\left(x^{(\alpha)} x^{\mu} y^{\lambda} D_{i}\right)
\end{aligned}
$$

and we conclude that $D_{\theta}+D_{\eta}=D_{\theta+\eta} \in \Omega$. Similarly, $k D_{\theta}=D_{k \theta} \in \Omega$ for any $k \in F$. Thus, $\Omega$ is a subspace of $\operatorname{der} \tilde{W}$.

To prove (ii), let $X$ be an arbitrary element of ad $\tilde{W} \cap \Omega$. Then there exist $B=\sum_{k=1}^{s} f_{k} D_{k} \in \tilde{W}$ and $\theta \in \Theta$ such that $X=\operatorname{ad} B=D_{\theta}$. Consequently,

$$
\operatorname{ad} B\left(D_{j}\right)=\left[\sum_{k=1}^{s} f_{k} D_{k}, D_{j}\right]=\sum_{k=1}^{s}(-1)^{\tilde{j} f f_{k} D_{k} \mid} D_{j}\left(f_{k}\right) D_{k}=D_{\theta}\left(D_{j}\right)=0
$$

for all $j \in Y$. Lemma 2.1 shows that $f_{k} \in G_{0}$ for all $k \in S$. Since $B \in \tilde{W}_{\overline{0}}$ by Lemma 4.8, we may assume that

$$
B=\sum_{k=1}^{n} f_{k} D_{k}+\sum_{k^{\prime}=r+1}^{s} f_{k^{\prime}} D_{k^{\prime}}
$$

Thus,

$$
\text { ad } B\left(x_{i} D_{i}+y_{t} D_{j}\right)=f_{i} D_{i}+f_{t} y_{t} D_{j}=D_{\theta}\left(x_{i} D_{i}+y_{t} D_{j}\right)=0
$$

for any $i \in Y_{0}, j \in Y_{1}, t \in Y_{2}$. This implies that $f_{i}=f_{t}=0$, whence $X=\operatorname{ad} B=0$. The proof is now complete.

Proposition 4.15. We have the equality of sets $\operatorname{der}_{-1} \tilde{W}=\operatorname{ad} \tilde{W}_{-1}$.

Proof. Let $\varphi \in \mathrm{h}\left(\operatorname{der}_{-1} \tilde{W}\right)$. We see that

$$
\tilde{W}_{0}=\operatorname{span}_{F}\left\{x_{i} D_{j}, x_{i} D_{i}, x_{i} y^{\lambda} D_{j}, x_{i} y^{\lambda} D_{i}, y^{\lambda} D_{l} \mid \lambda \in H, i, j \in Y, i \neq j, l \in Y_{2}\right\} .
$$

Clearly, ad $\tilde{W}_{-1} \subseteq \operatorname{der}_{-1} \tilde{W}$. It remains to show that ad $\tilde{W}_{-1} \supseteq \operatorname{der}_{-1} \tilde{W}$. We proceed in several steps.

Step 1. Let $\varphi\left(x_{i} D_{j}\right)=\sum_{k=1}^{r} a_{k} D_{k}$ and $\varphi\left(x_{h} D_{l}\right)=\sum_{k=1}^{r} b_{k} D_{k}$ for any $h, l \in Y \backslash\{i, j\}$, where $a_{k}, b_{k} \in G_{0}$. Since $\left[x_{i} D_{j}, x_{h} D_{l}\right]=0$, we see that

$$
\left[\sum_{k=1}^{r} a_{k} D_{k}, x_{h} D_{l}\right]+(-1)^{|\varphi|(\tilde{\imath}+\tilde{\jmath})}\left[x_{i} D_{j}, \sum_{k=1}^{r} b_{k} D_{k}\right]=0 .
$$


It follows that $a_{h} D_{l}-(-1)^{(\tilde{\imath}+\tilde{J})(|\varphi|+\tilde{l})} b_{i} D_{j}=0$. This means that $a_{h}=0$ for every $h \in$ $Y \backslash\{i, j\}$. Hence, $\varphi\left(x_{i} D_{j}\right)=a_{i} D_{i}+a_{j} D_{j}$.

Moreover, we may suppose that $\varphi\left(x_{i} D_{h}\right)=c_{i} D_{i}+c_{h} D_{h}$ and $\varphi\left(x_{h} D_{j}\right)=d_{h} D_{h}+d_{j} D_{j}$, where $c_{i}, c_{h}, d_{h}, d_{j} \in G_{0}$. Since $\left[x_{i} D_{h}, x_{h} D_{j}\right]=x_{i} D_{j}$, we see that

$$
\left[c_{i} D_{i}+c_{h} D_{h}, x_{h} D_{j}\right]+(-1)^{|\varphi|(\tilde{\imath}+\tilde{h})}\left[x_{i} D_{h}, d_{h} D_{h}+d_{j} D_{j}\right]=a_{i} D_{i}+a_{j} D_{j}
$$

It follows that $a_{i}=0$ and $\varphi\left(x_{i} D_{j}\right)=a_{j} D_{j}$.

In particular, suppose that $\varphi\left(x_{i} D_{i+1}\right)=h_{i} D_{i+1}$ for $i=1, \ldots, r-1$ and $\varphi\left(x_{r} D_{1}\right)=$ $h_{r} D_{1}$, where $h_{k} \in G_{0}$ for $k=1, \ldots, r$. Let $\psi=\varphi-\sum_{k=1}^{r} \operatorname{ad}\left(h_{k} D_{k}\right)$. Then

$$
\psi\left(x_{i} D_{i+1}\right)=\varphi\left(x_{i} D_{i+1}\right)-\sum_{k=1}^{r} \operatorname{ad}\left(h_{k} D_{k}\right)\left(x_{i} D_{i+1}\right)=h_{i} D_{i+1}-h_{i} D_{i+1}=0
$$

and $\psi\left(x_{r} D_{1}\right)=0$. In the following steps, we shall prove that $\psi\left(\tilde{W}_{0}\right)=0$.

Step 2. We claim that $\psi\left(x_{i} D_{j}\right)=0$. Indeed, if $i<j$, then by Step 1 we have

$$
\psi\left(x_{i} D_{i+2}\right)=\psi\left(\left[x_{i} D_{i+1}, x_{i+1} D_{i+2}\right]\right)=0
$$

and it follows that $\psi\left(x_{i} D_{j}\right)=0$. If $i>j$, then

$$
\psi\left(x_{r-1} D_{1}\right)=\psi\left(\left[x_{r-1} D_{r}, x_{r} D_{1}\right]\right)=0 .
$$

It follows that $\psi\left(x_{i} D_{1}\right)=0$. Consequently, $\psi\left(x_{i} D_{2}\right)=\psi\left(\left[x_{i} D_{1}, x_{1} D_{2}\right]\right)=0$ and it follows that $\psi\left(x_{i} D_{j}\right)=0$, establishing our claim.

Step 3. We claim that $\psi\left(x_{i} D_{i}\right)=0$. Suppose that $\psi\left(x_{i} D_{i}\right)=\sum_{k=1}^{r} e_{k} D_{k}$, where $e_{k} \in G_{0}$. Since $\left[x_{i} D_{i}, x_{j} D_{j+1}\right]=0$ for any $j \in Y \backslash\{i-1, i, r\}$, we see that

$$
\left[\sum_{k=1}^{r} e_{k} D_{k}, x_{j} D_{j+1}\right]=e_{j} D_{j+1}=0
$$

This implies that $e_{j}=0$. It follows that

$$
\psi\left(x_{i} D_{i}\right)=e_{i-1} D_{i-1}+e_{i} D_{i}+e_{r} D_{r} .
$$

Let $i \in Y \backslash\{1, r\}$. By applying $\psi$ to

$$
\left[x_{i} D_{i}, x_{i} D_{i+1}\right]=x_{i} D_{i+1}, \quad\left[x_{i} D_{i}, x_{i-1} D_{i}\right]=-x_{i-1} D_{i}, \quad\left[x_{i} D_{i}, x_{r} D_{1}\right]=0,
$$

we deduce that $e_{i}=e_{i-1}=e_{r}=0$. Hence, $\psi\left(x_{i} D_{i}\right)=0$ for any $i \in Y \backslash\{i, r\}$. We can similarly verify that $\psi\left(x_{1} D_{1}\right)=\psi\left(x_{r} D_{r}\right)=0$ and we have established our claim. 
Step 4. We claim that $\psi\left(x_{i} y^{\lambda} D_{j}\right)=0$. Suppose that $\psi\left(x_{i} y^{\lambda} D_{j}\right)=\sum_{k=1}^{r} f_{k} D_{k}$, where $f_{k} \in G_{0}$. Now Steps 2 and 3 imply that $\psi\left(x_{h} D_{l}\right)=0$. Since also $\left[x_{i} y^{\lambda} D_{j}, x_{h} D_{l}\right]=0$ for $h, l \in Y$ with $h \neq j$ and $l \neq i$, we deduce that

$$
\left[\sum_{k=1}^{r} f_{k} D_{k}, x_{h} D_{l}\right]=f_{h} D_{l}=0 .
$$

It follows that $f_{h}=0$ and $\psi\left(x_{i} y^{\lambda} D_{j}\right)=f_{j} D_{j}$. Since $\left[x_{i} D_{i}, x_{i} y^{\lambda} D_{j}\right]=x_{i} y^{\lambda} D_{j}$, we see that $0=\left[x_{i} D_{i}, f_{j} D_{j}\right]=f_{j} D_{j}$ by Step 3. It follows that $f_{j}=0$ and $\psi\left(x_{i} y^{\lambda} D_{j}\right)=0$, establishing our claim.

Step 5. We claim that $\psi\left(x_{i} y^{\lambda} D_{i}\right)=0$. Suppose that $\psi\left(x_{i} y^{\lambda} D_{i}\right)=\sum_{k=1}^{r} g_{k} D_{k}$, where $g_{k} \in G_{0}$. Since $\left[x_{i} y^{\lambda} D_{i}, x_{j} D_{j}\right]=0$ for any $j \in Y \backslash\{i\}$, we see that

$$
\left[\sum_{k=1}^{r} g_{k} D_{k}, x_{j} D_{j}\right]=g_{j} D_{j}=0 .
$$

It follows that $g_{j}=0$ and $\psi\left(x_{i} y^{\lambda} D_{i}\right)=g_{i} D_{i}$. Since

$$
\left[x_{i} y^{\lambda} D_{i}, x_{i} D_{j}\right]=x_{i} y^{\lambda} D_{j},
$$

we deduce that

$$
\left[g_{i} D_{i}, x_{i} D_{j}\right]=g_{i} D_{j}=0 .
$$

It follows that $g_{i}=0$ and $\psi\left(x_{i} y^{\lambda} D_{i}\right)=0$, establishing our claim.

Step 6. To complete the proof, we first show that $\psi\left(y^{\lambda} D_{l}\right)=0$. Let $\psi\left(y^{\lambda} D_{l}\right)=$ $\sum_{k=1}^{r} a_{k}^{\prime} D_{k}$, where $a_{k}^{\prime} \in G_{0}$. Since $\psi\left(x_{i} y^{-\lambda} D_{i}\right)=0$ for any $i \in Y$ by Step 5 , we may apply $\psi$ to

$$
\left[y^{\lambda} D_{l}, x_{i} y^{-\lambda} D_{i}\right]=-\lambda_{l} x_{i} D_{i}
$$

to deduce that $\left[\sum_{k=1}^{r} a_{k}^{\prime} D_{k}, x_{i} y^{-\lambda} D_{i}\right]=0$. It follows that $a_{i}^{\prime}=0$ and $\psi\left(y^{\lambda} D_{l}\right)=0$. From the discussion above, we conclude that $\psi\left(\tilde{W}_{0}\right)=0$. Thus, $\psi=0$ by Lemma 4.11 and $\operatorname{der}_{-1} \tilde{W}=\operatorname{ad} \tilde{W}_{-1}$.

We can use a similar method to that used to prove [15, Propositions 3 and 4] to deduce the following proposition.

Proposition 4.16. Let $t \in J$ and $t>1$. If there is no $k \in N$ such that $t=p^{k}$, then $\operatorname{der}_{-t} \tilde{W}=0$. If there exists $k \in N$ such that $t=p^{k}$, then

$$
\operatorname{der}_{-t} \tilde{W}=\operatorname{Span}_{G_{0}}\left\{\operatorname{ad} D_{i}^{t} \mid i \in Y_{0}\right\} .
$$

THEOREM 4.17. We have the equality

$$
\operatorname{der} \tilde{W}=\operatorname{ad} \tilde{W} \oplus \Omega \oplus \operatorname{Span}_{G_{0}}\left\{\left(\operatorname{ad} D_{i}\right)^{p^{k_{i}}} \mid i \in Y_{0}, 1 \leq k_{i}<t_{i}\right\} .
$$

Proof. This is a direct consequence of Propositions 4.12, 4.14, 4.15 and 4.16. 


\section{Acknowledgement}

The authors thank the referee for helpful suggestions.

\section{References}

[1] M. J. Celousov, 'Derivations of Lie algebras of Cartan-type', Izv. Vyssh. Uchebn. Zaved. Mat. 98 (1970), 126-134 (in Russian).

[2] R. Farnsteiner, 'Note on Frobenius extensions and restricted Lie superalgebras', J. Pure Appl. Algebra 108 (1996), 241-256.

[3] J.-Y. Fu, Q.-C. Zhang and C.-B. Jiang, 'The Cartan type modular Lie superalgebras KO', Comm. Algebra 34 (2006), 129-142.

[4] V. G. Kac, 'Lie superalgebras', Adv. Math. 98 (1977), 8-96.

[5] V. G. Kac, 'Classification of infinite-dimensional simple linearly compact Lie superalgebras', Adv. Math. 139 (1998), 1-55.

[6] Yu. Kochetkov and D. Leites, 'Simple Lie algebras in characteristic 2 recovered from superalgebras and on the notion of a simple finite group', Contemp. Math. 131 (1992), 59-67.

[7] W.-D. Liu, Y.-Z. Zhang and X.-L. Wang, 'The derivation algebra of the Cartan type Lie superalgebras HO', J. Algebra 273 (2004), 176-205.

[8] F.-M. Ma and Q.-C. Zhang, 'Derivation algebra of modular Lie superalgebra $K$ of Cartan type', J. Math. (Wuhan) 20 (2000), 431-435.

[9] V. M. Petrogradski, 'Identities in the enveloping algebras for modular Lie superalgebras', J. Algebra 145 (1992), 1-21.

[10] M. Scheunert, Theory of Lie Superalgebras, Lecture Notes in Mathematics, 716 (Springer, New York, 1979).

[11] H. Strade and R. Farnsteiner, Modular Lie Algebras and Their Representations, Monographs and Textbooks in Pure and Applied Mathematics, 116 (Marcel Dekker, New York, 1988).

[12] Y. Wang and Y.-Z. Zhang, 'Derivation algebra $\operatorname{Der}(H)$ and central extensions of Lie superalgebra', Comm. Algebra 32 (2004), 4117-4131.

[13] Y.-Z. Zhang, 'Finite-dimensional Lie superalgebras of Cartan-type over fields of prime characteristic', Chinese Sci. Bull. 42 (1997), 720-724.

[14] Y.-Z. Zhang and W.-D. Liu, Modular Lie Superalgebras (Science Press, Beijing, 2004), (in Chinese).

[15] Q.-C. Zhang and Y.-Z. Zhang, 'Derivation algebra of modular Lie superalgebras $W$ and $S$ of Cartan type', Acta Math. Sci. 20 (2000), 137-144.

YAN-QIN DONG, School of Mathematics and Statistics,

Northeast Normal University, Changchun 130024, Jilin, PR China

and

Aviation University of Air Force, Changchun 130022, Jilin, PR China

e-mail: dongyq384@nenu.edu.cn

YONG-ZHENG ZHANG, School of Mathematics and Statistics, Northeast Normal University, Changchun 130024, Jilin, PR China

e-mail: zhyz@nenu.edu.cn

ANGELO EBONZO, Transportation Management College, Dalian Maritime University, Dalian 116026, PR China

e-mail: angedan2000@yahoo.fr 